\section{Operating Room to Pediatric Intensive Care Unit Handoff: Improving Communication and Team Relations While Driving Process Improvement}

\author{
Sameer Shantaram Kamath ${ }^{1 *}$, Laurilyn Helmers ${ }^{2}$, Angela \\ Otto $^{3}$, Denise Kirk ${ }^{4}$, Jennifer Erdahl ${ }^{3}$ and Brian Wayling ${ }^{5}$
}

${ }^{1}$ Division of Critical Care, Duke Children's Hospital, Duke University Medical Center, Durham, NC, USA

${ }^{2}$ Department of Anesthesia, University of lowa Hospitals and Clinics, lowa, USA

${ }^{3}$ Department of Nursing (Pediatric Intensive Care Unit), University of lowa Hospitals and Clinics, lowa, USA

${ }^{4}$ Department of Nursing (Anesthesia), University of lowa Hospitals and Clinics, lowa, USA

${ }^{5}$ Tele Health Services, Intermountain Healthcare, Salt Lake City, UT, USA

\begin{abstract}
Background: The Operating Room (OR) to Pediatric Intensive Care Unit (PICU) handoff at University of lowa Hospital and Clinics was unstructured and inefficient with poor interdepartmental relations and communication.

Aim: Streamline the OR-to-PICU handoff process using the performance improvement principles of LEAN.

Hypothesis: A multidisciplinary project aimed at improving the OR-to-PICU handoff will result in improved efficiency, team dynamics and communication.

Method: The current handoff process was observed and recorded. Based on observations and staff survey results, a lean structured handoff process was designed which included a checklist. This proposed handoff was simulated, piloted and implemented after modifications. The new handoff was observed and recorded for comparison. The staff survey was repeated at 6,12 and 24 months to assess satisfaction and solicit feedback. Compliance with the structured handoff process was verified by auditing the checklist completed during handoff. Improvement in communication and
\end{abstract}

*Corresponding author: Sameer Shantaram Kamath, Division of Critical Care, Duke Children's Hospital, Duke University Medical Center, 2301 Erwin Road Durham, NC 27708, USA, Tel: +1 9196817692; Fax: +1 9196818357; E-mail: Sameer.kamath@duke.edu

Citation: Kamath SS, Helmers L, Otto A, Kirk D, Erdahl J, et al. (2016) Operating Room to Pediatric Intensive Care Unit Handoff: Improving Communication and Team Relations While Driving Process Improvement. J Anesth Clin Care 3: 017.

Received: January 28, 2016; Accepted: March 31, 2016; Published: April 15, 2016 interdepartmental relations was assessed via the survey questions and Patient Safety Net (PSN) reports.

Results: The structured handoff process needed fewer personnel and less time. Survey results suggested that communication, efficiency and interdepartmental relations were better with the new hand off process and results were consistent at 6,12 and 24 months. Average compliance with the structured handoff was $64 \%$ at 6 months, $56 \%$ at 12 months and $52 \%$ at 24 months, while the average compliance on day, night and weekend shifts was $50 \%$, $40 \%$ and $38 \%$ respectively. The number of PSN reports related to communication or professionalism decreased with the structured hand off process.

Conclusion: Multidisciplinary projects are a helpful way to drive process improvement while improving team dynamics and interdepartmental relations. The success of our project has resulted in the adoption of structured handoff for postoperative patients in critical care units across the institution.

Keywords: Communication; Handoff; Lean; Team; PICU

\section{Introduction}

Handoff is defined as the transfer of information, professional responsibility and accountability between individuals and teams [1]. The Pediatric Intensive Care Unit (PICU) is a high stress environment with potential for error resulting in patient harm. The Operating Room (OR) to PICU transfer represents a critical transition point in the continuum of care for children. The OR team performs several tasks during the handoff process such as patient transport, manual ventilation, titration of vasoactive medications and pain management. Lack of structured and rigorous handoff practices in these circumstances can result in adverse events due to errors of omission or commission [2].

In intensive care units, professionals from various disciplines interact closely on complex tasks essential to the support of critically ill patients. It is imperative for members of such a team to collaborate and work efficiently. Lack of mutual respect, unfamiliarity with each other's roles and unprofessional conduct can significantly hinder team performance and in turn affect quality of care [3]. A recent systematic review on postoperative handoff acknowledged the complexity of the process, importance of a structured and standardized approach, need for a multidisciplinary team participation and leadership engagement in the process [4].

At the University of Iowa Hospitals and Clinics (UIHC), the postoperative handoff in the PICU was not structured. The practice was idiosyncratic resulting in variability in information exchange and processes of care. The process was chaotic, with several inefficiencies and redundancies, resulting in frustration among team members that manifested itself into poor interdepartmental relations, staff communications, and underlying tension during patient care. Given these challenges, we decided to embark on a joint project to improve the OR-PICU handoff process. Our goals with this project were to provide the handoff process with structure and improve interdisciplinary professionalism. 


\section{Methods}

The PICU at UHIC is a twenty bed mixed medical and surgical unit and houses postoperative pediatric cardiac patients. We admit about 1000-1200 patients a year with a 60:40 split between surgical and medical admissions. Cardiac patients represented 19\% of our patients and accounted for about $27 \%$ of our bed days during the study period.

The desire to embark on this project was primarily driven by the need to improve communication between teams with the goal of improving patient safety. The authors Kamath SS, Helmers L and Otto A were the main champions of this multidisciplinary project. The division directors for pediatric anesthesia and pediatric critical care were the project sponsors. Given the complexity of the task at hand, we sought assistance from the UIHC operations excellence team, an internal group of engineers who practice LEAN/six sigma methodology to facilitate process mapping and performance improvement initiatives throughout the institution. One member of this team who is also one of the authors of this manuscript (BW) agreed to be the project coordinator. He spent a month observing the postoperative handoff process. This observation period was critical in understanding the complexities of the handoff process, team dynamics, and facilitated in process mapping. Based on his observations, he recommended using the principles of lean for this project. A detailed description of LEAN practices is beyond the scope of this manuscript; however the key methodology used was value stream mapping that examines the four key principles of flow, synchronization, levelling and pull. In our case, flow represents transition through various levels of care for a patient within an institution, synchronization refers to the harmony necessary to provide care to patients in a complex environment, levelling refers to presence of adequate resources and finally pull refers to readiness of the receiving team to accept responsibility for a patient from the team handing off care. The goal is identification of process waste and improving operational efficiencies [5].

In order to gain traction and acceptance for this initiative it was important to understand the problems and concerns perceived by staff [4]. We surveyed the PICU multidisciplinary team (physicians, nurses and respiratory therapists), OR team (physicians and nurse anesthetist's) and surgeons about the existing handoff process. (Survey 1) A structured handoff document was created and edited based on input from the PICU and OR teams to be used as a checklist during handoff (Figure 1). It was determined for simplicity and visibility to use a paper form instead of imbedding the checklist into the electronic medical record or another electronic tool. The handoff process was video recorded in October 2012 on a postoperative cardiac patient. Appropriate consent was obtained from involved staff as well as the patient family prior to filming. The video was audited for inefficiencies, errors and process waste by BW. Using the structured handoff tool. Personnel count and time taken for handoff were also noted from these videos as a surrogate for efficiency.

A process map was generated using the swim lane diagram (Figure 2) which served as a framework for our proposed ideal handoff process similar to Chen et al., [6]. The process map represented our ideal state while considering the existing flow of patients from the OR to the PICU. This was simulated and video recorded in November 2012. Again, this video was audited for errors, inefficiencies and process waste by BW. Staff education about the new handoff process was done by the project champions during

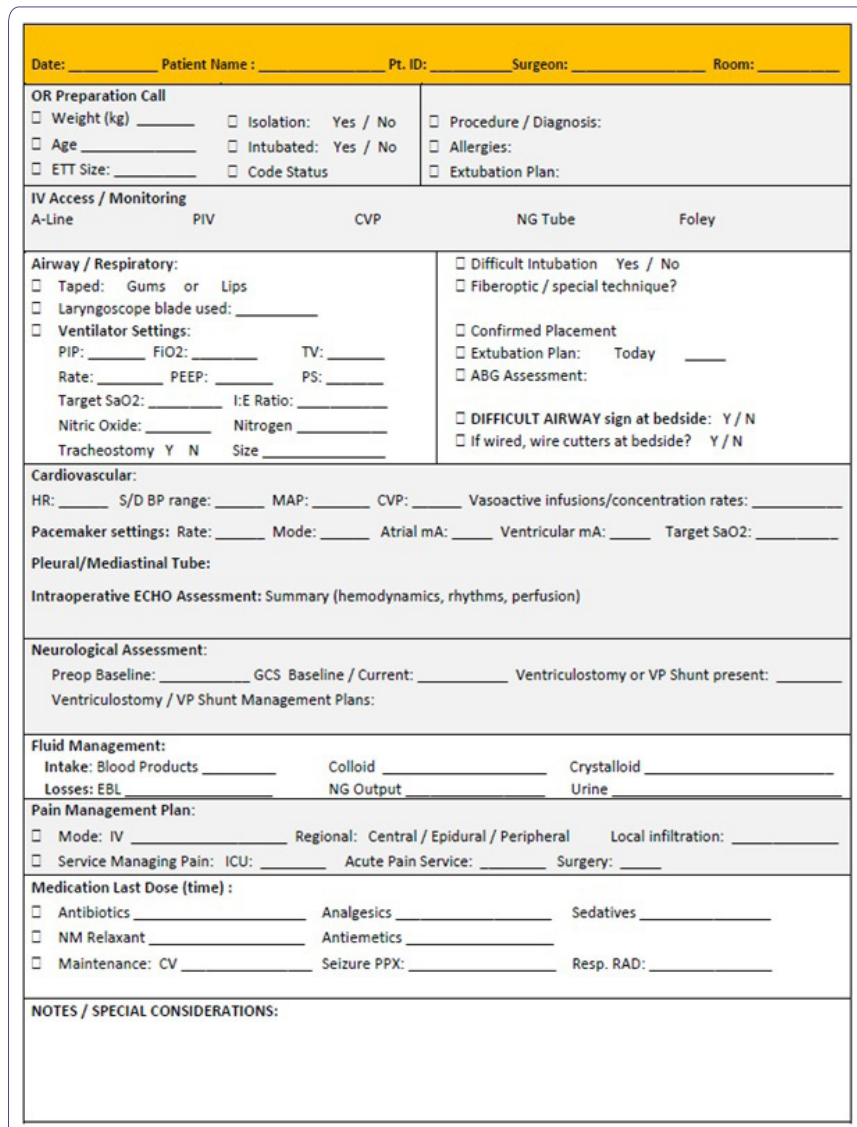

Figure 1: Handoff checklist tool.

staff meetings and at change of shift. Staff was able to view both the video recordings as well as data showing improved efficiency at these educational forums. After educating as many staff as possible in a 2 month period, we implemented the structured handoff process in February 2013.

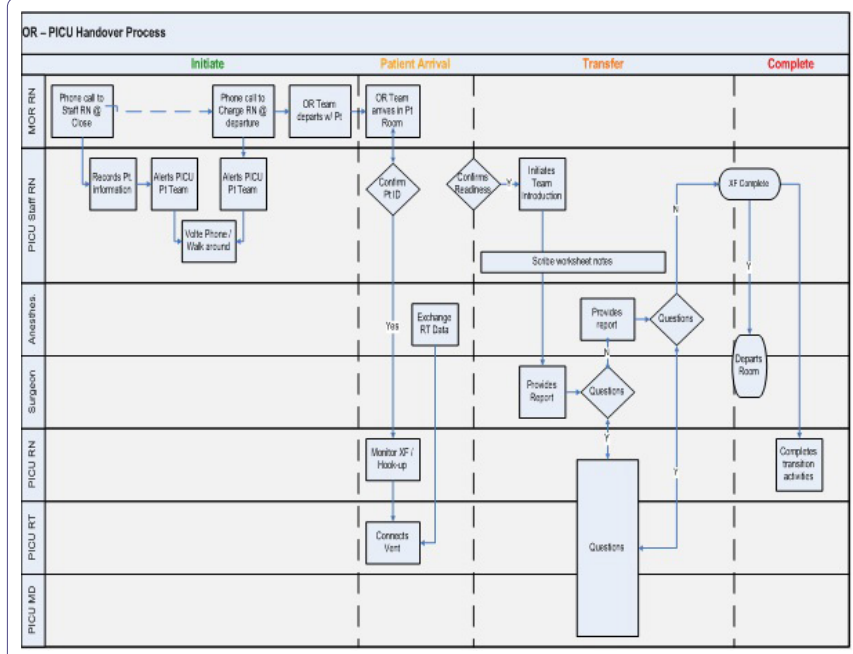

Figure 2: Swim lane diagram of proposed handoff Process map.

Staff survey was repeated after 6 months in September 2013 to obtain feedback and assess impact of the structured handoff process. (Survey 2) Compliance with the structured handoff process was audited by manually collecting the completed handoff sheets and cross checking those with number of postoperative admits we received on 


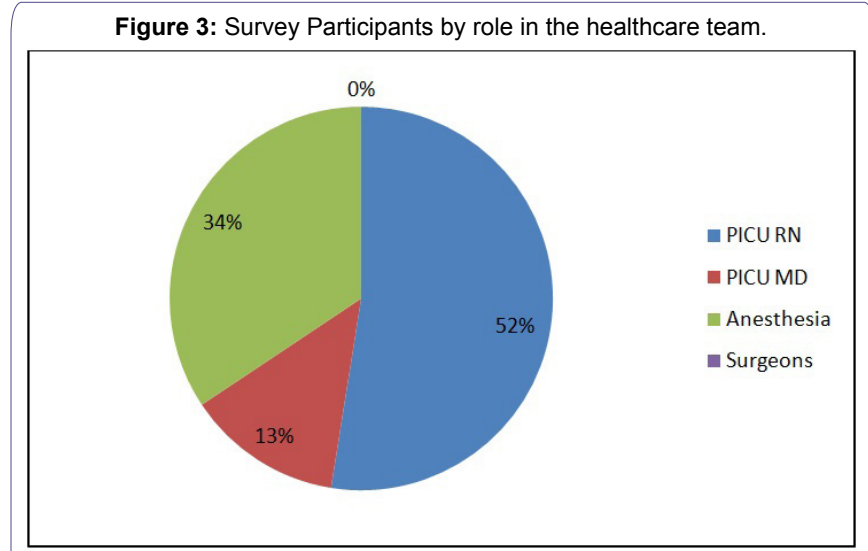

Figure 3a: Survey before implementation of structured handoff (Survey 1).

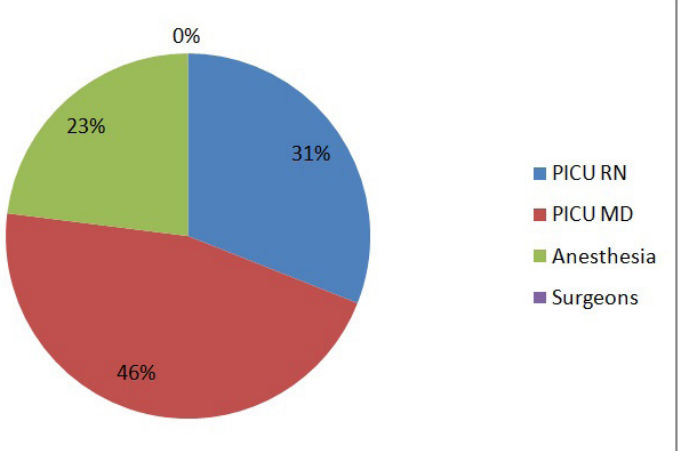

Figure 3b: Survey 6 months after implementation of structured handoff (Survey 2)

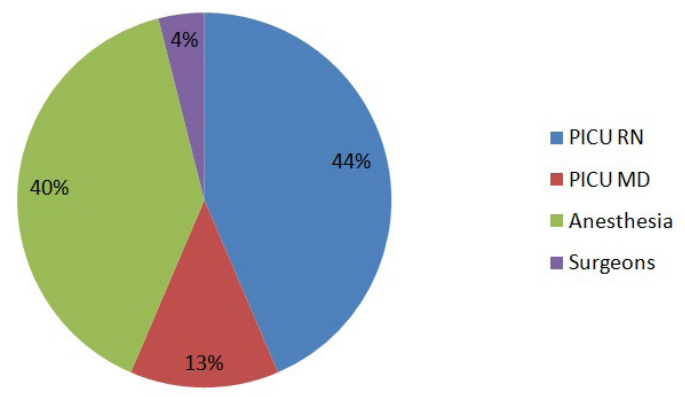

Figure 3c: Survey one year after implementation of structured handoff (Survey 3).

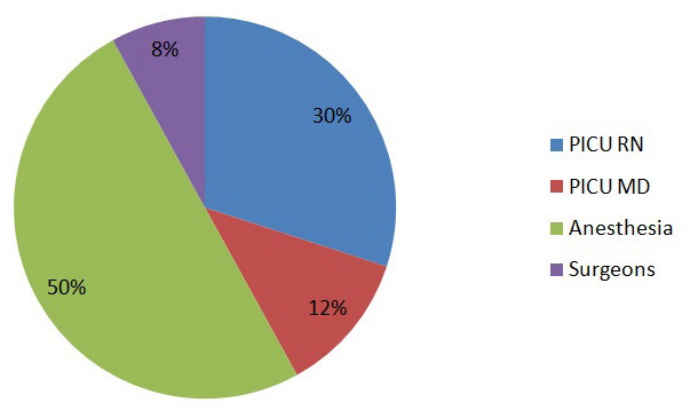

Figure 3d: Survey two years after implementation of structured handoff (Survey 4). a monthly basis by one of the authors (JE). If no handoff sheet was found for any patient we assumed a structured handoff did not happen. We did not audit these sheets or review charts to assess accuracy of information exchanged. The revised handoff process was video recorded as well and audited for inefficiencies, errors and process waste by BW. Finally, the staff survey was repeated one and two years after the project was launched in March 2014 (Survey 3) and 2015 (Survey 4) respectively to assess the impact of our intervention over time. These surveys were sent to the same group of providers each time. All the surveys were administered through the Qualtrics survey tool and were anonymous. Staff was given two reminders 2 weeks apart for each survey to maximize response rates. The only demographic data collected was their role in the healthcare team. Patient Safety Net (PSN) reports were audited for the duration of our study to identify reports related to errors, miscommunication or professionalism involving PICU and anesthesia.

\section{Results}

\section{Survey results}

Of the 270 participants surveyed 110 responded to survey 1 (40\%), 102 to survey 2 (38\%), 94 to survey 3 (34\%) and 90 to survey 4 (33\%). PICU nurses responded the most to survey 1 while anesthesia providers responded the most to surveys 3 and 4 . Surgeons were the lowest responders among health care personnel surveyed (Figure $3 \mathrm{a}-\mathrm{d}$ ).

The majority of responders expressed concerns about efficiency, process/flow and communication in the existent handoff process and indicated the need for a structured handoff process (Table 1). Communication, efficiency and interdepartmental relations were perceived to be better with the new handoff process, with those results staying consistent at 6,12 and 24 months after project implementation (Table 2). The percentage of responders who remained neutral decreased over time.

\section{Video audits}

The existent process was inefficient and the following were noted: 1) excessive staff count, 2) general confusion, 3) lack of clarity about leadership, 4) lack of a start and end point, 5) multiple simultaneous exchanges in silos, 6) inconsistent pre-arrival communications, and 7) missed critical elements. After the structured handoff process was implemented, the following observations were made: 1) limited number of essential staff present, 2) improved room dynamics, 3) a recognized leader for the handoff, 4) verbal triggers that clearly indicated start and stop point, 5) clarity in the information presented, and 6) improved critical data exchange. Existent handoff process involved 12-15 participants (range) for the postoperative cardiac patients was reduced to 6-8 (range) with the structured handoff. Average time taken to complete the handoff decreased from 12 minutes to 8 minutes ( $33 \%$ reduction).

\section{Compliance audit}

Average compliance with the structured handoff was $64 \%$ at 6 months, $56 \%$ at 12 months and $52 \%$ at 24 months post implementation (Figure 4a). Average compliance on day, night and weekend shifts was $50 \%, 40 \%$ and $38 \%$ respectively (Figure $4 \mathrm{~b}$ ). Compliance varied across different surgical service lines (Figure 4c). Compliance on pediatric surgical patients improved while that on ENT and neurosurgical patients remained steady over the two year period. 
Citation: Kamath SS, Helmers L, Otto A, Kirk D, Erdahl J, et al. (2016) Operating Room to Pediatric Intensive Care Unit Handoff: Improving Communication and Team Relations While Driving Process Improvement. J Anesth Clin Care 3: 017.

- Page 4 of 7 -

\begin{tabular}{|c|c|c|c|}
\hline Survey question & Strongly disagree or disagree & Strongly agree or agree & Neutral \\
\hline $\begin{array}{l}\text { The current handoff process for patient transfer of care to or } \\
\text { from the PICU is adequate and appropriate }\end{array}$ & $46.40 \%$ & $27.90 \%$ & $25.70 \%$ \\
\hline $\begin{array}{l}\text { A structured handoff will improve the quality and safety of } \\
\text { our patient care }\end{array}$ & $5.40 \%$ & $82.70 \%$ & $11.90 \%$ \\
\hline $\begin{array}{c}\text { Teamwork and good communication are evident in the } \\
\text { current handoff process }\end{array}$ & $30.90 \%$ & $34.60 \%$ & $34.50 \%$ \\
\hline $\begin{array}{l}\text { A structured handoff is needed for every patient going to or } \\
\text { coming back from the operating room. }\end{array}$ & $7.20 \%$ & $81.90 \%$ & $11.90 \%$ \\
\hline $\begin{array}{l}\text { A structured handoff will reduce confusion and improve } \\
\text { efficiency of all team members }\end{array}$ & $5.40 \%$ & $85.50 \%$ & $9.10 \%$ \\
\hline
\end{tabular}

Table 1: Results of survey before implementation of structured handoff (Survey1).

\begin{tabular}{|c|c|c|c|c|c|c|c|c|c|}
\hline \multirow{2}{*}{ Survey questions } & \multicolumn{3}{|c|}{ Strongly disagree or disagree } & \multicolumn{3}{|c|}{ Strongly Agree or Agree } & \multicolumn{3}{|c|}{ Neutral } \\
\hline & Survey 2 & Survey 3 & Survey 4 & Survey 2 & Survey 3 & Survey 4 & Survey 2 & Survey 3 & Survey 4 \\
\hline $\begin{array}{l}\text { The structured handoff process has } \\
\text { improved the quality and safety of our } \\
\text { patient care }\end{array}$ & $4 \%$ & $4 \%$ & $4 \%$ & $67 \%$ & $71 \%$ & $80 \%$ & $29 \%$ & $25 \%$ & $16 \%$ \\
\hline $\begin{array}{l}\text { Teamwork and good communication are } \\
\text { evident in a structured handoff process }\end{array}$ & $4 \%$ & $4 \%$ & $6 \%$ & $84 \%$ & $83 \%$ & $86 \%$ & $12 \%$ & $13 \%$ & $8 \%$ \\
\hline $\begin{array}{l}\text { The structured handoff has decreased } \\
\text { confusion and improved efficiency of all } \\
\text { team members }\end{array}$ & $7 \%$ & $3 \%$ & $4 \%$ & $64 \%$ & $82 \%$ & $84 \%$ & $29 \%$ & $15 \%$ & $12 \%$ \\
\hline $\begin{array}{l}\text { Structured handoff has improved inter- } \\
\text { disciplinary relationships }\end{array}$ & $5 \%$ & $6 \%$ & $4 \%$ & $68 \%$ & $60 \%$ & $80 \%$ & $27 \%$ & $34 \%$ & $16 \%$ \\
\hline $\begin{array}{l}\text { The structured handoff is an improve- } \\
\text { ment from previous methods }\end{array}$ & $7 \%$ & $4 \%$ & $4 \%$ & $79 \%$ & $86 \%$ & $84 \%$ & $14 \%$ & $10 \%$ & $12 \%$ \\
\hline $\begin{array}{c}\text { The structured handoff takes too much } \\
\text { time }\end{array}$ & $59 \%$ & $68 \%$ & $74 \%$ & $15 \%$ & $8 \%$ & $4 \%$ & $26 \%$ & $24 \%$ & $22 \%$ \\
\hline
\end{tabular}

Table 2: Results of surveys at 6 months (Survey 2), 1 year (Survey 3) and 2 years (Survey 4 ) post structured handoff implementation.

Compliance with orthopedic and cardiac patients dropped over the two year period.

\section{PSN report audit}

Number of PSN reports filed in relation to communication lapses or professionalism decreased post implementation of the structured hand off process ( 7 in the 2 years before to 1 in 24 months since implementation). No major errors were attributed to handoff before or after implementation of our project.

\section{Discussion}

With this multidisciplinary project we have demonstrated that the principles of LEAN can be used successfully to improve quality and efficiency of postoperative handoff. The structured handoff process was more efficient in that it took less time and fewer personnel to complete without jeopardizing patient safety. Staff perceived the structured handoff to be a significant improvement. Interdepartmental relations improved after implementation of the project. The results were consistent at 6,12 and 24 months after project implementation.

In our study the response rate for the surveys ranged from 30 and $40 \%$. This was despite three attempts (initial survey and 2 reminders) per survey made to solicit the most response. Although the overall response decreased from $40 \%$ for survey 1 to $30 \%$ for survey 4 , it was heartening to see an increase in participation from the anesthesia providers and surgeons over time. We believe that the success of the project may have improved participation of groups who were initially skeptical. A decrease in neutral responses with an increase in those favoring the new process also suggests general acceptance (Table 2). Kitch et al., in their study used a survey to assess adequacy of handoff [7]. This study however surveyed medical and surgical house staff concerning the quality and effects of handoff during care on the wards. Their survey was both paper and electronic with the paper format being administered after routine educational conferences for house staff from both departments. Our survey was in electronic format and distributed across disciplines (nursing, respiratory therapy, physicians and surgeons). The differences in study population and method of survey likely explain the significant difference in response rate between our experience and that of Kitch et al., more compelling efforts could have been made to improve survey response rates as in the Kitch et al., study; however we felt a 30-40\% response rate with representation across disciplines provided a reasonable sampling of staff perceptions and attitudes. Using other means to improve survey response rates given the multidisciplinary nature of the team was impractical.

In the ICU, patient care requires synchronization of efforts of highly skilled and specialized team members with diverse attitudes, skills and knowledge [8]. Divisive team communication can pose significant challenges to team performance. We noted a remarkable improvement in teamwork and interdepartmental relations following implementation of the structured handoff process. The perception of improved teamwork and relations improved from $60 \%$ to $80 \%$ over 
Figure 4: Compliance with structured handoff process by time of day (4a), by month (4b) and by surgical service (4c).

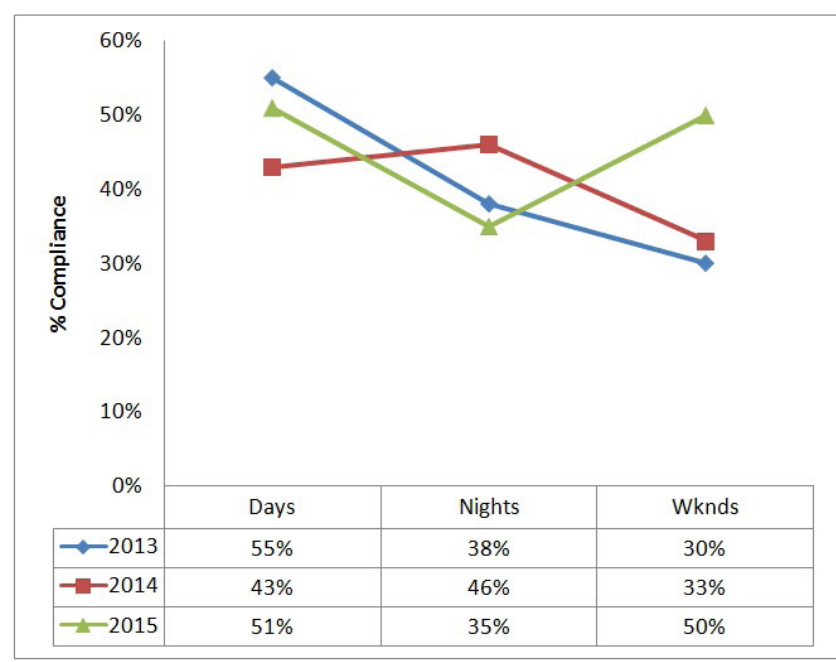

Figure 4a: Compliance by time of the day.

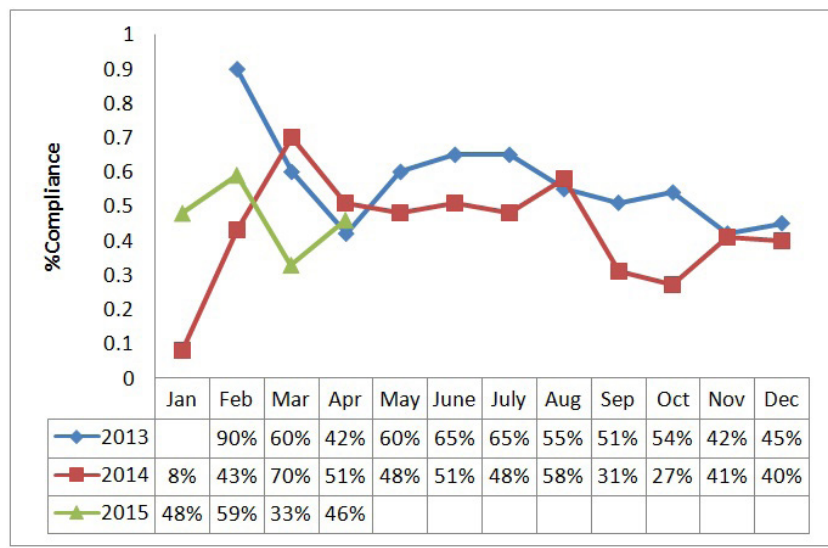

Figure 4b: Compliance by month.

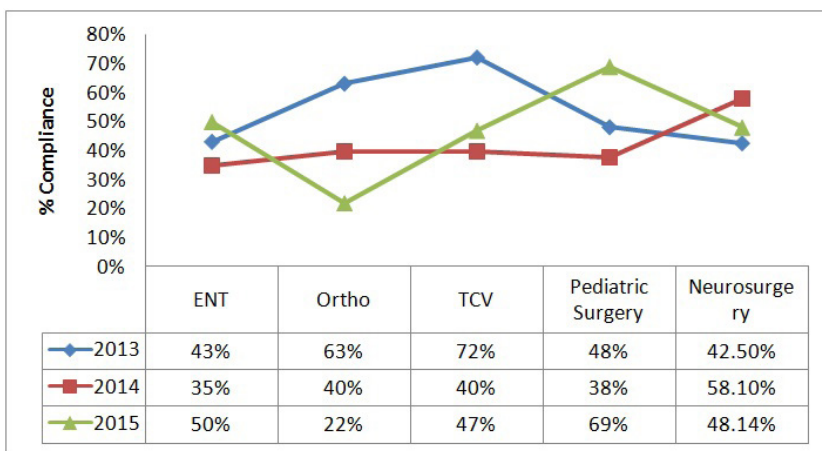

Figure 4c: Compliance by surgical service.

the two year period. Only one PSN related to professionalism was filed in the 2 year period after implementation of the project. Joy et al., in their study on handoff for post-operative pediatric cardiac patients also noted an improvement in team dynamics following implementation of structure to their handoff process similar to our experience [9]. We are hopeful that with continued adherence to the structured handoff process, the improved interdepartmental communication and relations will set the tone of professionalism with new generation of learners.
The post-operative cardiac patients represent a complex cohort of patients. Routinely they return from the OR in critical condition on mechanical ventilator support with multiple vasoactive infusions. There is a lot of information (preoperative, intraoperative and postoperative) that needs to be shared in addition to transitioning the patient from one set of monitors to another and exchanging support equipment. Minor errors in information exchange or care delivery can result in significant adverse events. We used a cardiac patient scenario to study current behaviors and model our ideal handoff process. We recognized that the handoff sheet, as designed, was most applicable for our cardiac patients, however, we consciously decided to use the same handoff sheet for all patient scenarios to limit variability. Reducing variability is an important quality improvement technique which is applied in other high risk and high complexity environments such as the PICU. Use of a structured handoff tool to improve the quality and safety of care for postoperative cardiac pediatric patients has been previously described by other investigators $[9,10]$. Breuer et al., in their paper described improved outcomes which were sustained over time through implementation of a standardized handover protocol in a multidisciplinary PICU [11]. They reported improved provider presence, improved communication, fewer antibiotic and analgesic delays or need for hemodynamic and respiratory interventions within 6 hours of PICU admission. To our knowledge, we are the first to use the same handoff tool uniformly across a mixed medical and surgical PICU that houses postoperative pediatric cardiac patients but did not measure the outcomes reported by Breuer et al. In their studies, Zavalkoff et al., and Joy et al., showed improvement in completeness of information exchanged without change in the duration of handoff in post-operative cardiac patients $[9,10]$. Their handoff episodes were observed and scored in real time for completeness of information exchanged. Our main focus was to impart structure to the handoff process and limit process waste rather that audit the quality and quantity of information exchanged. We thus are unable to comment on the quality or quantity of information exchanged. While Zavalkoff and Joy did not see a significant difference in time taken for the handoff, we noted a $33 \%$ decrease in time taken for handoff during both the simulation exercise and video recording of a follow up patient care scenario. Zavalkoff et al., noted a trend towards more patients being free from high risk events while Joy et al., showed a reduction in technical errors and handoff omissions in the post-intervention period. We did not observe these handoff events nor did we audit the handoff tool for completeness of information exchanged and are thus unable to comment on the same. At our institution any patient safety events including near misses are entered into a database (PSN). Any and all Healthcare Providers (HCP's) are encouraged to file such events on a voluntary basis. We used this database as a surrogate for significant misses or near misses for both time periods. There was no difference in number of PSN's either before or after implementation of the structured handoff process related to technical errors or high risk events.

Improving the efficiency of healthcare delivery is of utmost urgency. A lean handoff process frees up HCP's to attend to other equally critical tasks on the unit. We noted that the number of HCP's present during a handoff decreased from 12-15 to 6-8, an almost 50\% reduction, with the structured handoff. This finding was noted both during the simulation exercise and the recorded patient scenario. Time availability of HCP's is a precious resource and often in short supply. Optimizing the workforce helps to maximize impact, while mitigating fatigue and waste. We believe the improved efficiency in operations achieved with the structured handoff process will optimize 
resource utilization in our PICU and this to our knowledge has not been previously described.

Structured tools and checklists are known to improve processes in healthcare through standardization. We devised a structured handoff tool to facilitate exchange of critical information as described by Zavalkoff et al., and Joy et al., in their studies $[9,10]$. Postoperative cardiac patients are often on multiple infusions, have multiple lines in place and have a lot more information that needs to be exchanged between providers than other surgical patients. Given the multidisciplinary nature of our unit, we felt it was better to have a sheet that is usable for our busiest patient rather than miss any critical elements during handoff. Separate handoff sheets for cardiac and non-cardiac patients would likely result in confusion and hurt compliance with the process. Salzwedel et al., in a randomized controlled trial studying the effect of a checklist on the quality of post-anesthesia patient handoff also used video recording of handoffs which were then audited [12]. They studied handoff from the OR to the Post Anesthesia Care Unit (PACU) and noted an increase in time taken to complete the handoff contrary to our results but an improvement in the quality of information exchanged when a checklist was used as a guide. They video recorded all the study related handoffs while we recorded just a few handoff events (one before, one simulation and 3 case scenarios after). In our estimation, the videos were very powerful in illustrating the improved efficiency, team dynamics and organization. Our goal was to emphasize this efficiency to the team to improve acceptance of the new initiative. In our experience, the video's sent a powerful message and were worth a thousand words. Video recording all patient handovers would have been an excellent way to assess consistency and adequacy of information exchanged. It would have significantly strengthened our study but we did not have the resources to realistically accomplish that task.

We used completion of the handoff worksheet as a surrogate for compliance with the structured handoff process. Compliance with completion of the worksheet waned with time over 2 years, despite periodic reminders by project champions. While this may suggest that structured handoff did not occur almost half the time, it is more likely that the handoff occurred in a structured format but HCP's did not complete the worksheet or it was misplaced. Sustaining quality improvement initiatives that require behavioral changes is a huge challenge in any environment, and possibly more difficult in large academic institutions. Turnover of HCP's, including house staff and nursing staff, is the norm in academic healthcare and may have played a role in our suboptimal compliance rates. Some neurosurgical patients have their initial recovery in the PACU before admission to the PICU. They are not routinely accompanied by the anesthesiologist on admission, which may explain some missed opportunities in handoff in that group of patients. A modified process for those patients is being developed to facilitate a transfer of the information. We are unable to make any meaningful conclusions from data on compliance across surgical services but were reassured to see that the structured handoff process was not being preferentially used for cardiac patients where the handoff sheet was most applicable. Most of our elective surgical admits occur during the day, with late evening, night and weekend admits being primarily emergent cases. Compliance with the handoff process was lower on the night shift and on weekends when compared to the day shift. Typically in healthcare, resources are limited during these time periods and HCP's may be different on these shifts without the frequent education and re-education about new processes. This may explain the difference in compliance we noted between the various shifts.
Our study has several strengths. We engaged the operations excellence engineers and applied the principles of LEAN to this quality improvement initiative. We formed a multidisciplinary team to plan and implement the project to improve participation. We spent time in identifying the problem, soliciting feedback and planning the project before implementation. We video-recorded our old and new handoff process and shared these videos during educational sessions to convey a very compelling message. We have two years of follow-up data which suggests acceptance of the structured handoff process as the new norm. One weakness in this quality improvement project is the suboptimal survey response rates which may have skewed results towards those in favor of change. Another weakness would be our inability to audit the quality of the handoff, either via observation at the time of handoff or audit of the checklist completed; we lacked the resources to do either. Looking at these variables may have strengthened our study results. Our primary goal was the implementation of a structured handoff process to improve efficiency and interdepartmental relations which we achieved, as shown in our time studies and staff surveys, respectively.

In conclusion, through implementation of a multidisciplinary project aimed at improving postoperative handoff, we improved the efficiency of care while improving team dynamics. Principles of LEAN can be successfully applied for quality improvement in healthcare and the operations excellence team is an integral element in these institutional QI initiatives. The success of our pilot project has resulted in the adaptation of our model in many critical care environments across the institution. Utilizing continuous process improvement, we hope to improve compliance with the handoff process to $>80 \%$ during the day shift and $>60 \%$ during nights and weekends in the next academic year. We will continue to review the checklist to improve the information exchanged, the handoff completion times, and completion compliance.

\section{References}

1. Jeffcott SA, Evans SM, Cameron PA, Chin GS, Ibrahim JE (2009) Improving measurement in clinical handover. Qual Saf Health Care 18: 272-277.

2. Mistry KP, Landrigan CP, Goldmann DA, Bates DW (2005) Communication error during post-operative patient hand off in the pediatric intensive care unit.: 47. Crit Care Med 33: 12.

3. Mosser G, Begun JW (2014) Understanding Teamwork in Health Care. McGraw-Hill Education, New York, USA.

4. Møller TP, Madsen MD, Fuhrmann L, Østergaard D (2013) Postoperative handover: characteristics and considerations on improvement: a systematic review. Eur J Anaesthesiol 30: 229-242.

5. de Koning H, Verver JP, van den Heuvel J, Bisgaard S, Does RJ (2006) Lean six sigma in healthcare. J Healthc Qual 28: 4-11.

6. Chen JG, Wright MC, Smith PB, Jaggers J, Mistry KP (2011) Adaptation of a postoperative handoff communication process for children with heart disease: a quantitative study. Am J Med Qual 26: 380-386.

7. Kitch BT, Cooper JB, Zapol WM, Marder JE, Karson A, et al. (2008) Handoffs causing patient harm: a survey of medical and surgical house staff. Jt Comm J Qual Patient Saf 34: 563-570.

8. Hawryluck LA, Espin SL, Garwood KC, Evans CA, Lingard LA (2002) Pulling together and pushing apart: tides of tension in the ICU team. Acad Med 77: 73-76.

9. Joy BF, Elliott E, Hardy C, Sullivan C, Backer CL, et al. (2011) Standardized multidisciplinary protocol improves handover of cardiac surgery patients to the intensive care unit. Pediatr Crit Care Med 12: 304-308.

10. Zavalkoff SR, Razack SI, Lavoie J, Dancea AB (2011) Handover after pediatric heart surgery: a simple tool improves information exchange. Pediatr Crit Care Med 12: 309-313. 
Citation: Kamath SS, Helmers L, Otto A, Kirk D, Erdahl J, et al. (2016) Operating Room to Pediatric Intensive Care Unit Handoff: Improving Communication and Team Relations While Driving Process Improvement. J Anesth Clin Care 3: 017.

- Page 7 of 7 •

11. Breuer RK, Taicher B, Turner DA, Cheifetz IM, Rehder KJ (2015) Standardizing postoperative PICU handovers improves handover metrics and patient outcomes. Pediatr Crit Care Med 16: 256-263.
12. Salzwedel C, Bartz HJ, Kühnelt I, Appel D, Haupt O, et al. (2013) The effect of a checklist on the quality of post-anaesthesia patient handover: a randomized controlled trial. Int J Qual Health Care 25: 176-181. 